\title{
Effect of Intraoperative Mitomycin C Application in Dacryocystorhinostomy Surgery
}

\author{
Anurag Narula, Shilpa Singh and Vemparala Rajshekhar* \\ Department of Ophthalmology, India
}

*Corresponding author: Vemparala Rajshekhar, Department of Ophthalmology, New Delhi, India

Received: 䋊 May 30, 2019

Published: 眥June 06, 2019

\begin{abstract}
Background: The purpose of this study was to compare the success rate of conventional dacryocystorhinostomy (DCR), dacryocystorhinostomy with bicanalicular intubation and dacryocystorhinostomy with Mitomycin C(MMC).

Methods: A total of 50 patients having epiphora with nasolacrimal duct obstruction attending the eye Outpatients Department (OPD) of Attar Sain Jain Eye Hospital, Govt of NCT of Delhi between 2013 to 2015 were enrolled for the study and were equally randomized into two groups- conventional DCR and DCR with MMC. They were followed over a period of six months for studying improvement subjectively and objectively by syringing, Forced Dye Disappearance Test (FDDT) and Tear Meniscus Height (TMH).

Results: On subjective assessment, at the end of 180 days there were $5(20 \%)$ patients with watering in conventional group and $3(12 \%)$ patients in MMC group respectively. On objective assessment by syringing at the end of 180 days in the conventional group 5 patients (20\%) revealed non patency of the drainage system and in MMC group only 3 patients (12\%) showed non patency. At the end of study in the conventional group FDDT was positive in 16 eyes $(80 \%)$ and negative in 5 eyes (20\%) while in the MMC group FDDT was positive in 22 eyes (88\%) and negative in 3 eyes (12\%) Patients having TMH $<0.1 \mathrm{~mm}$ at the end of study were $11(44 \%)$ in conventional group while there were $22(75 \%)$ patients in MMC group.
\end{abstract}

Conclusion: On comparing conventional group bicanalicular intubation and Mitomycin $\mathrm{C}$ group it has been found that patients in the Mitomycin C group were more symptom free than conventional group, however difference was not statistically significant.

\section{Introduction}

Most patients who suffer from epiphora usually have a blockage located near the end of the system within the nasolacrimal duct. The aim of DCR surgery is to create a new bypass channel so that the tears can pass from the lacrimal sac into the nose again through the new bypass channel, therefore bypassing the blockage within the nasolacrimal duct However, the chance of success varies from patient to patient and it is about $60-90 \%$ [1] and is dependent on various factors including type of obstruction and site of obstruction, patient symptoms and any previous surgery [2]. It has been found that two most frequent causes of DCR failure are closure of the osteotomy site by fibroproliferative changes and obstruction of the common canaliculus [3]. Therefore, different methods have been tried to improve success rate of DCR surgery like making larger osteum intraoperatively [4]. A small length of soft plastic(silicon) tubing can also be used through the bypass channel, to help maintain patency of the new drainage system while the new bypass channel heals. This is easily removed in the outpatient clinic shortly after the operation (usually within 3 months) [5].
We know that Mitomycin C (MMC), an anticancer agent isolated from Streptomyces caespitosus, can significantly suppress fibrosis and vascular ingrowth after exposure to the filtration site of the trabeculectomy for glaucoma [6]. In DCR surgery, we tried to use MMC soaking over the osteotomy site and the anastomosed flaps to suppress fibrous proliferation and scar formation. Theoretically, this modification should reduce the fibrous adhesion between the osteotomy site and the nasal septum as well as inhibit scarring around the opening of the common canaliculus. Thus, MMC should prevent further shrinkage of the final surface area of the osteotomy and prevent the obstruction of the common canaliculus opening. So, the use of Mitomycin C(MMC) intraoperatively at the osteotomy site should decrease the failure rate and the cost of operation and postoperative discomfort seen in patients with bicanalicular intubation [7].

\section{Materials and Methods}

The study was conducted in Department of Ophthalmology Attar Sain Jain Eye and General Hospital, Government of NCT of 
Delhi. Total 50 patients with NLD block with epiphora attending the Out Patients Department(OPD) in Ophthalmology department were randomly assigned into three groups of 25 each and followed over period of 6 months to see for success and failure of the procedure, as usually patients start appearing 1- 3 months post op and almost all cases of failure report within 6 months.
a) Group A-Conventional Dacrocystorhinostomy (DCR)
b) Group B- DCR with Mitomycin C(MMC)

Patients were assessed preoperatively with detail history regarding age of patient, onset and duration of symptoms, unilateral or bilateral, intermittent or constant, pain, discharge, or swelling, trauma, operations or medication. Meticulous history was taken to rule out exclusion criteria like bleeding tendencies, coagulation abnormalities, Nasal problems and failed previous similar surgeries. Full ophthalmic and ENT examination of all patients was done (Table 1).

Table 1: Criteria for Inclusion and Exclusion.

\begin{tabular}{|l|l|}
\hline Inclusion criteria & \multicolumn{1}{|c|}{ Patients having NLD blocks on syringing } \\
\hline & All other sites of lacrimal passage block except \\
NLD block \\
Exclusion criteria & Age $<12$ yrs \\
& Pregnant Women \\
& Nasal Pathology including severe DNS \\
\hline
\end{tabular}

\section{Investigations}

a) Syringing and probing was done to rule out canalicular obstruction and for confirmation of nasolacrimal duct obstruction

b) Fluorescein dye disappearance test

The patients were randomized into above mentioned three groups. The patients were followed up for 6 months as per the protocol. Subjective and objective findings were noted.

The patients were assessed subjectively by asking about severity of watering and graded into three categories-
a) Symptom free
b) Symptomatically improved and
c) No improvement

Objective assessment was done by
A. Syringing
i. Patent
ii. Non patent
B. Tear meniscus height (TMH)
i. $\quad<0.1 \mathrm{~mm}$
ii. $\quad 0.1-0.2 \mathrm{~mm} \mathrm{i}$
iii. $>0.2 \mathrm{~mm}$
C. Fluorescein dye disappearance test (FDDT)
i. $\quad$ Positive FDDT
ii. Negative FD

\section{Surgery Techniques}

a) Conventional DCR: The procedure was essentially the same except that we have kept osteotomy opening $12 \times 15 \mathrm{~mm}$ in each case to avoid confounding error (Table 2).

Table 2: Subjective and objective assessment in conventional group.

\begin{tabular}{|c|c|c|c|c|}
\hline \multicolumn{5}{|c|}{ CONVENTIONAL GROUP (N=25) } \\
\hline & Day 7 & Day 60 & Day 90 & Day 180 \\
\hline 1.Symptom Free & $5(20 \%)$ & $14(56 \%)$ & $13(52 \%)$ & $12(48 \%)$ \\
\hline 2.Improved & $18(72 \%)$ & $8(32 \%)$ & $7(28 \%)$ & $8(32 \%)$ \\
\hline $\begin{array}{c}\text { 3. NO } \\
\text { Improvement }\end{array}$ & $2(8 \%)$ & $3(12 \%)$ & $5(20 \%)$ & $5(20 \%)$ \\
\hline \multicolumn{5}{|c|}{ B. OBJECTIVE ASSESSMENT SYRINGING } \\
\hline \multicolumn{5}{|c|}{ II) TMH } \\
\hline a) Patent & $23(92 \%)$ & $13(52 \%)$ & $13(52 \%)$ & $13(52 \%)$ \\
\hline b) Non-Patent & $2(8 \%)$ & $2(8 \%)$ & $5(20 \%)$ & $5(20 \%)$ \\
\hline \multicolumn{5}{|c|}{ III) FDDT } \\
\hline a) <0.1mm & $5(20 \%)$ & $18(72 \%)$ & $12(48 \%)$ & $11(44 \%)$ \\
\hline b)0.1-0.2mm & $18(72 \%)$ & $9(36 \%)$ & $8(32 \%)$ & $9(36 \%)$ \\
\hline c)>0.2mm & $18(72 \%)$ & $3(12 \%)$ & $5(20 \%)$ & $5(20 \%)$ \\
\hline \multicolumn{5}{|c|}{ Im) } \\
\hline a) Positive & $23(92 \%)$ & $22(88 \%)$ & $20(80 \%)$ & $20(80 \%)$ \\
\hline b) Negative & $2(8 \%)$ & $3(12 \%)$ & $5(20 \%)$ & $5(20 \%)$ \\
\hline
\end{tabular}

Table 3: Subjective and objective assessment in MMC group.

\begin{tabular}{|c|c|c|c|c|}
\hline \multicolumn{5}{|c|}{ MMC GROUP (N=25) } \\
\hline & Day 7 & Day 60 & Day 90 & Day 60 \\
\hline 1.Symptom Free & $6(24 \%)$ & $16(64 \%)$ & $17(68 \%)$ & $17(68 \%)$ \\
\hline 2.Improved & $18(72 \%)$ & $6(36 \%)$ & $5(20 \%)$ & $5(20 \%)$ \\
\hline $\begin{array}{c}\text { 3. NO } \\
\text { Improvement }\end{array}$ & $1(4 \%)$ & $3(12 \%)$ & $3(12 \%)$ & $3(12 \%)$ \\
\hline \multicolumn{5}{|c|}{ B. OBJECTIVE ASSESSMENT } \\
\hline \multicolumn{5}{|c|}{ II)TMH TMINGING } \\
\hline a) Patent & $24(96 \%)$ & $22(88 \%)$ & $22(88 \%)$ & $22(88 \%)$ \\
\hline b) Non-Patent & $1(4 \%)$ & $3(12 \%)$ & $3(12 \%)$ & $3(12 \%)$ \\
\hline \multicolumn{5}{|c|}{ III)FDDT } \\
\hline a) <0.1mm & $7(28 \%)$ & $14(56 \%)$ & $15(60 \%)$ & $15(60 \%)$ \\
\hline b)0.1-0.2mm & $17(68 \%)$ & $8(32 \%)$ & $7(28 \%)$ & $7(28 \%)$ \\
\hline c)>0.2mm & $1(4 \%)$ & $3(12 \%)$ & $3(12 \%)$ & $3(12 \%)$ \\
\hline \multicolumn{5}{|c|}{ (1m) } \\
\hline a) Positive & $24(96 \%)$ & $22(88 \%)$ & $22(88 \%)$ & $22(88 \%)$ \\
\hline b) Negative & $1(4 \%)$ & $3(12 \%)$ & $3(12 \%)$ & $3(12 \%)$ \\
\hline
\end{tabular}


b) DCR with MMC: In this group only modification done was application of MMC $0.2 \mathrm{mg} / \mathrm{ml}$ at the osteotomy site for $10 \mathrm{~min}$ taking care not to apply on the skin and then washed thoroughly with saline other steps were similar like conventional DCR. Any complication during surgery was noted (Table 3).

\section{Visits}

On post-operative day 1 wound condition, nasal bleeding, syringing (except Group B) and any subjective symptoms were noted on 7th post-operative day in addition to above findings FDDT and height of tear meniscus is also noted. Further follow ups were done on days $30,60,90$ and 180 with above mentioned criteria. On day 90 tube removal done in Group B.

\section{Results}

\section{Age Distribution}

The mean age in the conventional group (group A) was 40.28 years and 38.76 years in the MMC group (group B). The mean ages of the patients were comparable in the two groups.

There was no significant statistical difference in age between the two groups- paired t-test values between groups is 0.67 (on comparing all groups-t test, $\mathrm{P}>0.05$ ).

\section{Sex Distribution}

The 50 patients included in the study comprised of 22 male patients and the remaining 28 being female patients. They were evenly distributed among the study groups. There was no significant statistical difference in sex distribution between groups- paired T-test values between group is 0.97 (on comparing all groups- $t$ test, $\mathrm{P}>0.05$ ). As we followed the patients over a period of 180 days we found that the assessment of subjective symptoms shows improvement in all groups at the 60 day visit and Thereafter from period 60 to 180 days there was increase in the number of symptom free patients in MMC group (group b); but those in conventional group (group A) showed a decline in symptom free patients (from 14 to 12 patients) and an increase in no improvement group ( from 2 to 5 patients). At the end of 180 days there were 5 (20\%) patients with watering in conventional and 3(12\%) in MMC group. Although, intraoperative MMC application seemed to improve the subjective symptoms reported by patients, the satisfaction rates did not differ significantly between the groups $\mathrm{P}=0.717$ (two sided), Pearson Chi-square with likelihood ratio 0.716

\section{Discussion}

It was observed in our study that the subjective symptoms improved in all the groups, but the maximum improvement occurred in the MMC group. The maximum improvement in all three groups was seen during the initial period of 60 days. This improvement was maintained throughout the 180 days of the follow-up in the MMC group. On the other hand, in the conventional group the symptoms reappeared from 60 to 180 -day period. Similarly, the objective findings showed comparable improvement in all three groups; which remained constant in the MMC group but worsened in the conventional group (group A) on prolonged follow up, from 60 to 90 day. These encouraging findings, in the MMC group, can be attributed to the prevention of fibrosis around the osteotomy site. These finding can be compared with the study conducted by Yeatts et al. [8] and Lio et al. [9] who showed higher symptomatic improvement in MMC group as compared to conventional group.

It has been observed, that over a period of time worsening tend to occur around 2-3 months, probably due to fibrosis of osteotomy site. These findings were reported in literature by Mcpherson SD et al. [10] and Pico G et al. [11] who showed common cause of failure was dense fibrous tissue at the osteotomy site. Lindberg JV et al. [12] also showed that osteotomy opening

made during surgery usually narrow down due to fibrosis. In our study, on the 60th and 90th post-operative day of follow-up, there were worsening of the subjective symptoms and objective evaluations; more so in the conventional group (group A) as compared to other group most probably due to fibrosis at the osteotomy site. All these results showed that MMC augmented DCR are better as compared to conventional DCR, when compared in terms of subjective and objective improvement and also in terms of longevity of the procedures. However, when statistical methods were applied no statistically significant differences were seen. This could be explained by the less number of patients 25 in each group. However more patients could not be accommodated as this was a surgical study having time constraints. During the early postoperative period the patients in all the three groups had complaints of watering, probably attributable to lid edema and inflammation at the operative site. However, during further follow ups there was marked improvement in the subjective symptoms.

Although, intraoperative MMC application seemed to improve the subjective symptoms reported by patients, the satisfaction rates did not differ significantly between the three groups. These finding were consistent with the result of the studies conducted by Yeatts et al. [8] and Cem Yildirim et al. [13], who have showed that satisfaction and success rates of MMC group were higher than those of the control group however the differences were not statistically significant. Many complications such as corneal ulcer, corneal perforations, scleral calcification, secondary cataract, endophthalmitis, hypotony and maculopathy have been reported with the use of MMC in both pterygium and glaucoma filtration surgeries [14,15]. In our study, wound gaping was observed in two patients in MMC group. The wound gaping was sutured in the operating room. After one week, one of these patients developed fistulous opening at the lacrimal sac area. He underwent fistulectomy and suturing of the wound. This patient was having fistulous tract preoperatively. On the other hand, no wound gaping was seen in conventional group (group A). Higher rate of wound gaping in MMC group was probably due to accidental application of MMC to the skin incision site and these complications have been reported in the literature [9]. However, side effects like mucosal necrosis or infection were not noted in any of the patients during the six months follow up. No nasal or gastrointestinal irritation had been observed during intraoperative MMC application.

In conclusion, in the present study the satisfaction and success rates of the EXT- DCR with MMC application were found to be higher 
than those of standard EXT- DCR, however the differences did not reach statistical significance. No deleterious effect was noted with MMC treatment. Although the sample size and power of the study were too small to make definite statements, intraoperative MMC application seems to be a safe adjuvant that could help to increase the success rate of EXT-DCR surgery in primary acquired nasolacrimal duct obstruction. Application of a wound-healing inhibitor is a new treatment modality in EXT-DCR surgery and more studies with larger series must be done on this topic before one can make further conclusions. A rationale for the duration and method of MMC application should also be determined. A rationale for the duration and method of MMC application should also be determined.

\section{References}

1. Walland MJ, Rose GE (1994) Factors effecting the success rate of open lacrimal sac surgery. Br J Ophthalmol 78: 888-891.

2. Tarbet KJ, Custer PL (1995) External dacryocystorhinostomy: surgical success, patient satisfaction and economic cost. Ophthalmology 102: $1065-1070$

3. Mclachlan DL, Shannon GM, Flangen JC (1980) Results of dacryocystorhinostomy: Analysis of reoperations. Ophthalmic surgery 11: 427-430.

4. Argin A, Görür K, Ozcan C, Arslan E, Ozmen C, et al. (2008) The role of larger osteotomy in long term success in external dacryocystorhinostomy. J Plast Reconstr Aesthet Surg 61(6): 615-619.

5. Fulcher T, O Connor M, Moriarty P (1998) Nasolacrimal intubation in adults. Br J Ophthalmol 82(9): 1039-1041.
6. Lee DA, Lee TC, Cortes AE, Kitada (1990) Effect of Mithramycin Mitomycin, Daunorubicin and Bleomycin on human subconjuctival fibroblast attachment and proliferation. Invest Ophthalos Vis Sci 31(10): 2136-2144.

7. Veloudios A, Harvey JT, Philippon M (1991) Long-term placement of silastic nasolacrimal tubes. Ophthalmic Surg 22(4): 225-227.

8. RP Yeatts, RB Neves (1999) Use of Mitomycin C in repeat dacryocystorhinostomy. Ophthalmic Plastic and Reconstructive Surgery 15(1): 19-22.

9. Shu L Liao, Shine CS Kao. JHS Tseng (2000) Results of intraoperative Mitomycin-C application in dacryocystorhinostomy. Br J Ophthalmol 84(8): 903-906.

10. McPherson SD, Egelston D (1956) Dacryocystorhinostomy: a review of 106 operation. Am J Ophthalmol 47(3): 328-331.

11. Picó G (1971) A modified technique of external dacryocystorhinostomy. Am J Ophthalmol 72(4): 679-690.

12. Linberg JV, Anderson RL, Bumsted RM, et al. (1982) study of intranasal ostium external dacryocystorhinostomy. Arch Opthalmol 100(11): 1758-1762.

13. Cem Yildirim, Volkan Yaylali, Arif Esme, Serap Ozden (2007) Long-term results of adjunctive use of Mitomycin $\mathrm{C}$ in external dacryocystorhinostomy. Int Ophthalmol 27(1): 31-35.

14. Bergstrom TJ, Wilkinson WS, Skuta GL, et al. (1991) The effects of subconjunctival Mitomycin-C on glaucoma filtration surgery in rabbits. Arch Ophthalmol 109(12): 1725-1730.

15. Rubinfeld RS, Pfister RR, Stein RM, et al. (1992) Serious complications of topical Mitomycin-C after pterygium surgery. Ophthalmology 99(11): $1647-1654$.
(C) (P) This work is licensed under Creative

To Submit Your Article Click Here: $\quad$ Submit Article

DOI: $10.32474 /$ TOOAJ.2019.02.000136

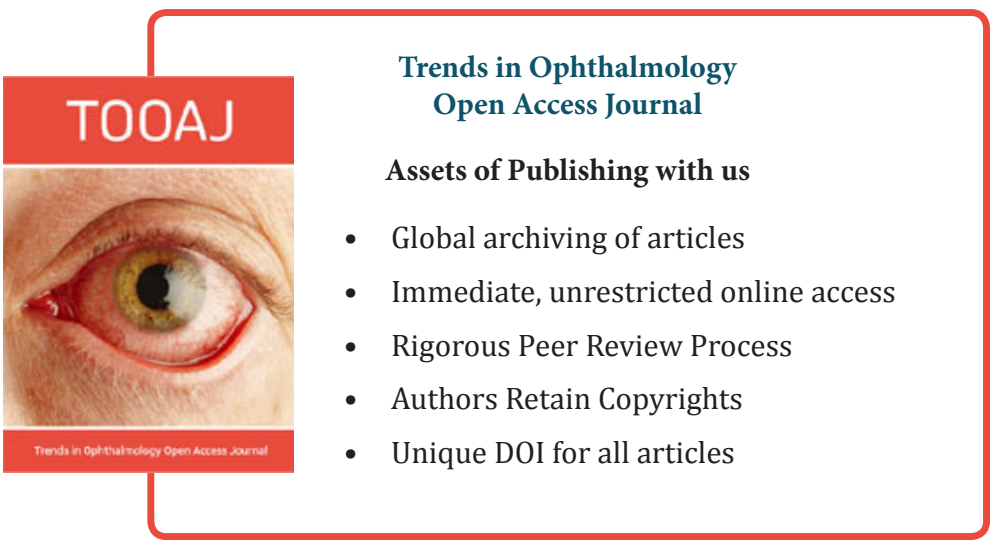

